\title{
Detection of antimicrobial resistant Salmonella enterica strains in samples of ground hedgehogs (Atelerix albiventris) reared as pets in the urban area of Santiago, Chile
}

\author{
Siboney Perez ${ }^{\mathrm{a}}$, Marlen Barreto ${ }^{\mathrm{b}}$, Patricio Retamal ${ }^{\mathrm{a}}$
}

\begin{abstract}
The breeding of exotic pets has become a popular practice in Chile and, within this group of animals, small mammals such as guinea pigs and hedgehogs have gained importance due to their docile behaviour. The most common exotic hedgehog species in Chile is the African pygmy hedgehog (Atelerix albiventris). It has been reported that these pets are reservoirs of some zoonotic pathogens, among which Salmonella enterica constitutes an important threat for the owners. This study aimed to detect the presence of Salmonella strains in faeces from hedgehogs (Atelerix albiventris) admitted to a veterinary clinic in Santiago, Chile, and to characterise the antimicrobial susceptibility of the isolated strains. From 200 animals sampled, S. enterica was detected in 5 hedgehogs, corresponding to serotypes Muenchen (2), Infantis (2) and IV43:z4,z23:- (1). Furthermore, phenotypic antimicrobial resistance was determined in all subsp. enterica isolates. These results suggest that in Chile these exotic pets constitute a potential hazard for public health, therefore, supporting educational campaigns about basic biosecurity measures is necessary, mostly aimed at pet owners and risk groups.

Key words: hedgehogs, Salmonella, antimicrobial resistance, Chile.
\end{abstract}

\section{INTRODUCTION}

Non-traditional pets have become very popular in developed and developing countries. Apart from exotic reptiles, amphibians and fishes, unconventional pets include a variety of species of mammals, such as nonhuman primates, ferrets, prairie dogs, and hedgehogs (Hoelzer et al 2011).

Hedgehogs are native to Europe, Asia and Africa, and include multiple species, although two of them are generally reared as pets: the European hedgehog (Erinaceus europaeus), and the African hedgehog (Atelerix spp. and Hemiechinus spp.) (Riley and Chomel 2005). These animals are omnivores, with a diet composed of insects, slugs, worms, snails, fungi, fruits, and certain vegetables like spinaches, carrots and broccoli (Santana et al 2010). Among the hedgehogs, A. albiventris is becoming the most popular pet in Chile and around the world, with increasing populations in captivity (Fredes and Román 2004, Okada et al 2018). Since 2007, the importation of these animals to Chile has not been allowed and all the individuals legally commercialised have been bred in the country. However, there is no record of the reproduction, number and distribution of these animals housed as pets.

With regard to the risks associated with their breeding, some studies have determined that these mammals could act as hosts of zoonotic pathogens, including Salmonella

Received: 01.07.2020.

Accepted: 19.01.2021.

aFacultad de Ciencias Veterinarias y Pecuarias, Universidad de Chile, Santiago, Chile.

b'Instituto de Ciencias Biomédicas, Universidad Autónoma de Chile, Santiago, Chile.

*Corresponding author: P Retamal, Av. Sta Rosa 11735, La Pintana, 8820808, Santiago, Chile; pretamal@uchile.cl spp., Yersinia spp. and Mycobacterium spp., among others (Riley and Chomel 2005, Santana et al 2010, Keeble and Koterwas 2020). Salmonella enterica is an enteric pathogen widely distributed in nature, which causes infection in a range of hosts including humans and other mammals, birds and reptiles (Marus et al 2019). More than 2,600 serotypes have been described within this species, including some host-restricted and other host-generalist serotypes. When it comes to zoonotic infections, Salmonella serotypes are commonly transmitted by consumption of contaminated animal and plant-derived food. This usually results in self-limiting gastroenteritis (Ferrari et al 2019), although patients with some risk factors, such as infants, immunocompromised individuals, and the elderly have shown extra-intestinal infections that can cause meningitis, sepsis and even death (Gordon 2008). In the last 30 years, infection with this bacterium in humans accounts for the most common notifiable outbreaks of any infectious disease (Smith et al 2014). In Chile, official records show a similar trend in which Salmonella represents the major cause of foodborne disease outbreaks among cases with a confirmed diagnosis (Olea et al 2012).

In non-outbreak, individual and domestic contexts of non-typhoidal Salmonella infections, other transmission pathways may lead to disease in humans, such as the direct or indirect exposure to pets (Younus et al 2010, Braun et al 2015, MacDonald et al 2018). These cases appear sporadically and it mostly affects only a few people, being less studied and reported, and therefore constituting much more common events than is believed (Boore et al 2015).

Hedgehogs in captivity represent a public health risk due to the epidemiological evidence that links bacterial detection in these animals, which generally appear as asymptomatic carriers with a high Salmonella load, with a disease in humans (Riley and Chomel 2005, Kagambega et al 2013, Anderson et al 2017, MacDonald et al 2018). 
Also, antimicrobial resistant phenotypes have been described in pathogens isolated from the gastrointestinal tract of hedgehogs, generating concern about the potential transmission of these genetic traits to the gut microbiota of owners (Zare and Ghorbani-Choboghlo 2015).

The objective of this work was to report the isolation of zoonotic Salmonella serotypes in African pygmy hedgehogs (A. albiventrix) kept as pets in the Metropolitan Region from Chile, and characterise antimicrobial-resistance phenotypes in these isolates.

\section{MATERIAL AND METHODS}

\section{SAMPLES}

Between March 2017 and July 2018, 200 animals (103 males and 97 females) were sampled upon admission to a veterinary clinic specialising in exotic animals, located in the urban area of the city of Santiago, Chile. This sample size represented $10 \%$ of all hedgehogs registered in the clinic. For sampling, animals were weighted and maintained in individual cages until they defecated. Then, fresh faeces were immediately collected with sterile swabs, immersed into Cary Blair transport medium and stored at $4{ }^{\circ} \mathrm{C}$ for analyses at the laboratory (Fresno et al 2013). The age was registered from clinical records or by directly asking to owners. Each animal was sampled only once.

The inclusion criteria corresponded to animals that had not been treated with antimicrobials two weeks prior to sampling. Before the sampling procedure, their owners signed an informed consent that had been approved by the Bioethics Committee (14-2017 VETUCH) of the Faculty of Veterinary and Animal Sciences, University of Chile.

\section{BACTERIOLOGICAL ISOLATION}

Once at the laboratory, faeces were processed as reported previously (Fresno et al 2013). Briefly, swabs were inoculated into $5 \mathrm{~mL}$ of buffered peptone water (Difco®) supplemented with $20 \mathrm{mg} / \mathrm{mL}$ of Novobiocin (Sigma®) (Jensen et al 2003) and then incubated at $37^{\circ} \mathrm{C}$ for 24 hours. Later, $100 \mu \mathrm{L}$ of the bacterial suspension was seeded into Rappaport Vassilidis semisolid agar (MSRV, Oxoid®) supplemented with $20 \mathrm{mg} / \mathrm{mL}$ of Novobiocin, and incubated for $24-48$ hours at $41.5^{\circ} \mathrm{C}$. Cultures with bacterial growth were plated in Xylosa Lysine Deoxycolate agar (XLD, Difco $\left.{ }^{\circledR}\right)$ and incubated at $37^{\circ} \mathrm{C}$ for 24 hours. Suspicious colonies of Salmonella spp. were confirmed by the detection of the invA gene in a PCR test (Malorny et al. 2003) and then were serotyped at the Chilean Institute of Public Health, the national reference laboratory, according to the Kauffman-White scheme ${ }^{1}$.

1 Grimont PA, Weill FX. 2007. Antigenic formulae of the Salmonella serovars. $9^{\text {th }}$ ed. WHO Collaborating Center for Reference and Research on Salmonella, Institut Pasteur, Paris, France. Available at https://www.pasteur.fr/sites/default/files/veng_0.pdf.

\section{ANTIBIOTIC RESISTANCE PHENOTYPES}

The antimicrobial susceptibility was evaluated by the disk diffusion method (Kirby Bauer), according to the standards recommended by the Clinical Laboratory Standards Institute (CLSI 2012) and the National Antimicrobial Resistance Monitoring System (NARMS 2011). The following antimicrobials, which are of frequent use in veterinary and human medicine, were evaluated: enrofloxacin $(10 \mu \mathrm{g})$, amoxicillin + clavulanic acid $(20 / 10 \mu g)$, tetracycline $(30 \mu \mathrm{g})$, ampicillin $(10 \mu \mathrm{g})$, cefadroxil $(30 \mu \mathrm{g})$, azithromycin $(15 \mu \mathrm{g})$, ceftriaxone $(30 \mu \mathrm{g})$, kanamycin $(30 \mu \mathrm{g})$, nalidixic acid $(30 \mu \mathrm{g})$, streptomycin $(10 \mu \mathrm{g})$, ceftiofur. Escherichia coli ATCC 25922 was used as a control strain. The multidrug resistance (MDR) condition was determined by the simultaneous resistance to three or more antimicrobial classes.

\section{ANALYSES OF RESULTS}

Associations between bacteriological results and data from hosts (sex, weight, and age) were explored with ANOVA and categorical data analyses using Infostat (v2010) software.

\section{RESULTS AND DISCUSSION}

From 200 samples analysed, a total of 5 animals resulted positive for $(2.5 \%) S$. enterica isolation in their faeces. These detections included subspecies enterica, with serotypes Muenchen (2) and Infantis (2), and subspecies houtenae, with the serotype 43:z4,z23:- (1). All S. enterica subsp. enterica strains showed antimicrobial resistance phenotypes, including a multi-drug resistant $S$. enterica ser. Muenchen isolate (table 1). In contrast, the S. enterica subsp. houtenae isolate was pansusceptible. No associations $(P>0.05)$ were detected between the variables analysed.

The results obtained from the study confirm the presence of antimicrobial resistant $S$. enterica strains in hedgehogs, representing potential risk for owners in Chile. Studies from other countries have reported infections by Salmonella spp. in humans who kept hedgehogs as pets ${ }^{2}$ (Riley and Chomel 2005, Chomel et al 2007). Although there is no evidence of similar cases in Chile, it is possible that animal to human transmission is occurring. In general, such zoonotic events are underreported since the disease generally appears individually rather than collectively in the exposed population and also because of the self-limiting nature of the disease (Morse et al 2012).

In the last years, $S$. enterica ser. Muenchen has been related to foodborne outbreaks linked to turtle meat, pigs and plant-derived foods in Australia, Europe and North America (Jackson et al 2013, Bonardi et al 2016, Draper

2 CDC. 2019. Outbreak of Salmonella infections linked to pet hedgehogs. Available at https://www.cdc.gov/salmonella/typhimurium-09-20/ index.html. 
Table 1. Description of Salmonella enterica isolates, animal hosts data, and antimicrobial resistance phenotypes.

\begin{tabular}{|c|c|c|c|c|c|c|c|c|c|c|c|c|c|c|c|}
\hline \multirow{2}{*}{ Salmonella serotype } & \multirow{2}{*}{ Sex } & \multirow{2}{*}{$\begin{array}{c}\text { Weight } \\
\text { (gr) }\end{array}$} & \multirow{2}{*}{ Age } & \multicolumn{12}{|c|}{ Antimicrobial resistance* } \\
\hline & & & & $\mathrm{AMC}$ & AMP & TE & CFR & EFT & ENR & $\mathrm{CN}$ & SXT & AZN & $\mathrm{CRO}$ & $\mathrm{K}$ & NA \\
\hline Muenchen & $\mathrm{F}$ & 231 & $3 \mathrm{~m}$ & - & - & - & I & - & - & - & - & - & - & - & - \\
\hline Muenchen & M & 302 & $1.8 \mathrm{y}$ & - & $\mathrm{R}$ & $\mathrm{R}$ & - & $\mathrm{R}$ & - & - & - & - & - & - & - \\
\hline Infantis & $\mathrm{F}$ & 304 & $2.2 \mathrm{y}$ & - & - & - & - & $\mathrm{R}$ & - & - & - & - & - & - & - \\
\hline Infantis & $\mathrm{F}$ & 365 & $4.2 \mathrm{y}$ & - & - & - & I & - & - & - & - & - & - & - & - \\
\hline IV 43: z4, z23:- & $\mathrm{F}$ & 348 & $2.7 \mathrm{y}$ & - & - & - & - & - & - & - & - & - & - & - & - \\
\hline
\end{tabular}

*AMC, Amoxicillin/ Clavulanic acid; AMP, Ampicillin; TE, Tetracycline; CFR, Cefadroxil; EFT, Ceftiofur; ENR, enrofloxacin; CN, Gentamicin; SXT, Sulfamethoxazole /Trimethoprim; AZN, Azithromicin; CRO, Ceftriaxone; CIP, Ciprofloxacin; K, Kanamycin; NA, Nalidixic acid. Underlined and non-underlined abbreviations correspond to critically important and highly important antimicrobials, respectively (WHO 2019). R, Resistant, I, Intermediate, - Susceptible.

et al 2017, Schielke et al 2017), respectively. These reports suggest a diversity of sources when causing disease in humans. This contrasts with the other serotype found, S. enterica ser. Infantis, which is an emerging pathogen generally associated to outbreaks by consumption of poultry products (Aviv et al 2019). The last isolate, the IV 43: z4, z23:- serotype, belongs to the subsp. houtenae, and has been mostly detected in reptiles. Despite its lower pathogenic potential when compared with subsp. enterica serotypes, extra-intestinal infections in children have been described (Lamas et al 2018).

The specific transmission pathways by which hedgehogs from this study resulted colonised with different Salmonella serotypes are unknown. They might become infected in pet stores or breeders, or after being sold, due to co-habitation with other pets or by consumption of contaminated food offered by their owners to these mammals. Furthermore, the access to other kind of food or preys, is also frequent, especially in some household systems with a partial confinement. In these situations, the potential consumption of insects, lizards or other commodities and animals, could allow their contamination with diverse Salmonella serotypes, as has been described in this study and elsewhere (Kagambega et al 2013).

Antimicrobial resistance is an emerging threat to public health at a global scale, in which major drivers for selection of resistant agents are the misuse and overuse of antimicrobials, both in the human and animal context (Millanao et al 2018). In this regard, the World Health Organization categorised these drugs according to their therapeutic relevance, trying to preserve their effectiveness for human medicine. In this study, we found resistance phenotypes against critically important (ampicillin and ceftiofur) and highly important antimicrobials (tetracycline) (WHO 2019) in both Muenchen and Infantis serotypes (table 1). The resistance against ceftiofur is of particular concern, because the bacterium could be potentially carrying the plasmid encoded blaCMY-2 gene, that also confers resistance against ceftriaxone (Alcaine et al 2005), an antimicrobial recommended for the treatment of extraintestinal infections of Salmonella in humans (Yang et al 2016). Such bacterial traits might be circumstantially detected in hedgehogs after the consumption of contaminated food or may represent the in vivo selection of resistant clones because of antimicrobial-based therapies used in these animals. Although our exclusion criteria for sampling was the absence of treatments of this type during the last two weeks prior to sampling, persistent infections with drugresistant bacteria might also be occurring (Pignon and Mayer 2011). Whatever the source of pathogens in pets, a zoonotic transmission to people, especially young children, the elderly and immunocompromised patients (Lawson et al 2018), may have severe clinical outcomes (Anderson et al 2017), especially in extra-intestinal infections which require an antibiotic-based therapy (Ichimi et al 2018). The pet hedgehog ownership is not recommended in these risk groups (Keeble and Koterwas 2020).

Non-specific clinical signs of salmonellosis have been reported in hedgehogs, such as anorexia, diarrhea and weight loss (Riley and Chomel 2005, Keeble and Koterwas 2020). However, all positive animals detected in this work were asymptomatic, a condition that predominates in previous reports (Craig et al 1997, Riley and Chomel 2005, Santana et al 2010, Hoelzer et al 2011, Lawson et al 2018), suggesting that sampling of animals in order to detect Salmonella carriers should not be constrained by their clinical status. In this regard, owners must be aware about risks presented by the handle of apparently healthy animals. Some basic biosecurity measures could be recommended by veterinarians to hedgehog owners in the context of veterinary practice, such as washing hands after touching them or cleaning its enclosures and supervising that children play safely, avoiding kisses and snuggles with these animals ${ }^{1}$.

This work constitutes the first description of Salmonella infection in hedgehogs from South America. In other regions, a range of $S$. enterica prevalence fluctuating between 0\% (Handeland et al 2002) and 96\% (Kagambega 
et al 2013) has been reported both in free-living and domesticated hedgehogs. A $2.5 \%$ infection rate detected in pets sampled for this study suggests a low exposure to Salmonella that probably represents adequate management in most cases of domestic ownership. However, hedgehogs may shed Salmonella spp. intermittently, especially during exposure to stress from inadequate nutrition, housing and care, as has been reported in other pets and livestock (Hoelzer et al 2011). In this study we sampled each animal only once, and bacterial infection data may be underestimated. Other considerations regarding our results could be the limitations in the diagnostic sensitivity of isolation procedures, and the unknown number of hedgehogs and husbandry conditions of these animals living in Chile.

Finally, the presence of multidrug resistant Salmonella strains was confirmed in hedgehogs in Chile. Further studies should address possible animal to human transmission events, which have been documented several times in other regions. The zoonotic risk and the impacts on natural ecosystems if these animals are released, are major factors to be considered when establishing public policies for their pet ownership.

\section{ACKNOWLEDGEMENTS}

The authors would like to thank the collaborating personnel from the Exzootic Vet Veterinary Clinic. This research received financial support from the Fondecyt project No. 11110398.

\section{CONFLICT OF INTEREST}

The authors do not have any conflict of interest.

\section{REFERENCES}

Alcaine S, Sukhnanand S, Warnick L, Su W, McGann P, et al. 2005. Ceftiofur-resistant Salmonella strains isolated from dairy farms represent multiple widely distributed subtypes that evolved by independent horizontal gene transfer. Antimicrob Agents Chemother 49, 4061-4067.

Anderson TC, Marsden-Haug N, Morris JF, Culpepper W, Bessette N, et al. 2017. Multistate outbreak of human Salmonella typhimurium infections linked to pet hedgehogs - United States, 2011-2013. Zoonoses Public Health 64, 290-298.

Aviv G, Cornelius A, Davidovich M, Cohen H, Suwand A, et al. 2019. The emerging Salmonella Infantis expresses lower levels of SPI-1 genes and causes milder colitis in mice and lower rates of invasive disease in humans than Salmonella typhimurium. J Infect Dis 220, 1071-1081.

Bonardi S, Alpigiani I, Bruini I, Barilli E, Brindani F, et al. 2016. Detection of Salmonella enterica in pigs at slaughter and comparison with human isolates in Italy. Int J Food Microbiol 218, 44-50.

Boore AL, Hoekstra RM, Iwamoto M, Fields PI, Bishop RD, et al. 2015. Salmonella enterica infections in the United States and assessment of coefficients of variation: A novel approach to identify epidemiologic characteristics of individual serotypes, 1996-2011. PLoS One 10, e0145416.

Braun S, Spalloni W, Ferreccio F, Postigo J, Fernández A, et al. 2015. Salmonella spp. gastroenteritis associated to pet turtles in three infants. Rev Chil Infectol 32, 334-338.
Chomel BB, Belotto A, Meslin FX. 2007. Wildlife, exotic pets, and emerging zoonoses. Emerg Infect Dis 13, 6-11.

Craig C, Styliadis S, Woodward D, Werker D. 1997. African pygmy hedgehog-associated Salmonella tilene in Canada. Can Commun Dis Rep 23, 129-131; discussion 131-122.

Draper ADK, James CL, Pascall JE, Shield KJ, Langrell J, et al. 2017. An outbreak of Salmonella Muenchen after consuming sea turtle, Northern Territory, Australia, 2017. Commun Dis Intell Q Rep 41, e290-e294.

Ferrari RG, Rosario DKA, Cunha-Neto A, Mano SB, Figueiredo EES, et al. 2019. Worldwide epidemiology of Salmonella serovars in animal-based foods: a meta-analysis. Appl Environ Microbiol 85, e00591- e00519.

Fredes F, Román D. 2004. Fauna parasitaria en erizos de tierra africanos (Atelerix albiventris). Parasitol latinoam 59, 79-81.

Fresno M, Barrera V, Gornall V, Lillo P, Paredes N, et al. 2013. Identification of diverse Salmonella serotypes, virulotypes, and antimicrobial resistance phenotypes in waterfowl from Chile. Vector Borne Zoonotic Dis 13, 884-887.

Gordon MA. 2008. Salmonella infections in immunocompromised adults. $J$ Infect 56, 413-422.

Handeland K, Refsum T, Johansen BS, Holstad G, Knutsen G, et al. 2002. Prevalence of Salmonella typhimurium infection in Norwegian hedgehog populations associated with two human disease outbreaks. Epidemiol Infect 128, 523-527.

Hoelzer K, Moreno Switt AI, Wiedmann M. 2011. Animal contact as a source of human non-typhoidal salmonellosis. Vet Res 42, 34.

Ichimi R, Yoshino A, Higashigawa M. 2018. Salmonella stanley bacteremia transmitted from a pet hedgehog. Pediatr Int 60, 606-607.

Jackson BR, Griffin PM, Cole D, Walsh KA, Chai SJ. 2013. Outbreakassociated Salmonella enterica serotypes and food Commodities, United States, 1998-2008. Emerg Infect Dis 19, 1239-1244.

Jensen AN, Sørensen G, Baggesen DL, Bødker R, Hoorfar J. 2003. Addition of Novobiocin in pre-enrichment step can improve Salmonella culture protocol of modified semisolid Rappaport-Vassiliadis. J Microbiol Methods 55, 249-55.

Kagambega A, Lienemann T, Aulu L, Traore AS, Barro N, et al. 2013. Prevalence and characterization of Salmonella enterica from the feces of cattle, poultry, swine and hedgehogs in Burkina Faso and their comparison to human Salmonella isolates. BMC Microbiol 13, 253.

Keeble E, Koterwas B. 2020. Salmonellosis in Hedgehogs. Vet Clin North Am Exot Anim Pract 23, 459-470.

Lamas A, Miranda JM, Regal P, Vázquez B, Franco CM, et al. 2018. A comprehensive review of non-enterica subspecies of Salmonella enterica. Microbiol Res 206, 60-73.

Lawson B, Franklinos LHV, Rodriguez-Ramos Fernandez J, Wend-Hansen C, Nair S, et al. 2018. Salmonella enteritidis ST183: emerging and endemic biotypes affecting western European hedgehogs (Erinaceus europaeus) and people in Great Britain. Sci Rep 8, 2449.

MacDonald E, White R, Mexia R, Bruun T, Kapperud G, et al. 2019. The role of domestic reservoirs in domestically acquired Salmonella infections in Norway: epidemiology of salmonellosis, 2000-2015, and results of a national prospective case-control study, 2010-2012. Epidemiol Infect 147, e43.

Malorny B, Hoorfar J, Bunge C, Helmuth R. 2003. Multicenter validation of the analytical accuracy of Salmonella PCR: towards an international standard. Appl Environ Microbiol 69, 290-296.

Marus JR, Magee MJ, Manikonda K, Nichols MC. 2019. Outbreaks of Salmonella enterica infections linked to animal contact: Demographic and outbreak characteristics and comparison to foodborne outbreaks-United States, 2009-2014. Zoonoses Public Health 66, 370-376.

Millanao A, Barrientos-Schaffeld C, Siegel-Tike C, Tomova A, Ivanova L, et al. 2018. Antimicrobial resistance in Chile and the one health paradigm: Dealing with threats to human and veterinary health resulting from antimicrobial use in salmon aquaculture and the clinic. Rev Chil Infectol 35, 299-308 
Morse SS, Mazet JA, Woolhouse M, Parrish CR, Carroll D, et al. 2012. Prediction and prevention of the next pandemic zoonosis. Lancet 380, 1956-1965.

Okada K, Kondo H, Sumi A, Kagawa Y. 2018. A retrospective study of disease incidence in African pygmy hedgehogs (Atelerix albiventris). J Vet Med Sci 80, 1504-1510.

Olea A, Díaz J, Fuentes R, Vaquero A, García M. 2012. Vigilancia de brotes de enfermedades transmitidas por alimentos en Chile. Rev Chil Infectol 29, 504-510

Pignon C, Mayer J. 2011. Zoonoses of ferrets, hedgehogs, and sugar gliders. Vet Clin North Am Exot Anim Pract 14, 533-549.

Riley PY, Chomel BB. 2005. Hedgehog zoonoses. Emerg Infect Dis 11, 1-5.

Santana EM, Jantz HE, Best TL. 2010. Atelerix albiventris (Erinaceomorpha: Erinaceidae). Mamm Species 42, 99-110.

Schielke A, Rabsch W, Prager R, Simon S, Fruth A, et al. 2017. Two consecutive large outbreaks of Salmonella Muenchen linked to pig farming in Germany, 2013 to 2014: Is something missing in our regulatory framework? Euro Surveill 22, 30528.
Smith KF, Goldberg M, Rosenthal S, Carlson L, Chen J, et al. 2014. Global rise in human infectious disease outbreaks. J R Soc Interface $11,20140950$.

Yang W, Chan O, Wu T, Chen C, Su L, et al. 2016. Development of ceftriaxone resistance in Salmonella enterica serotype Oranienburg during therapy for bacteremia. J Microbiol Immunol Infect 49, 41-45.

Younus M, Wilkins MJ, Davies HD, Rahbar MH, Funk J, et al. 2010. The role of exposures to animals and other risk factors in sporadic, non-typhoidal Salmonella infections in Michigan children. Zoonoses Public Health 57, e170-e176.

WHO. 2019. Critically important antimicrobials for human medicine. WHO, Geneva, Switzerland.

Zare P, Ghorbani-Choboghlo H. 2015. Isolation and characterization of multidrug-resistant gram-negative bacteria found in free-ranging long-eared hedgehogs (Erinaceus concolor) from Tabriz, Iran. J Exot Pet Med 24, 235-239. 
